\title{
Numerical Solution Method of Nonlinear Guided Modes with a Finite Difference Complex Axis Beam Propagation Method
}

\author{
Frank Wijnands, Hugo J. W. M. Hoekstra, Gijs J. M. Krijnen, Member, IEEE, and René M. de Ridder
}

\begin{abstract}
A method to construct modal fields for an arbitrary one- or two-dimensional intensity dependent refractive index structure is described. An arbitrary starting field is propagated along an imaginary axis using the Finite Difference Beam Propagation Method (FDBPM) based upon the Slowly Varying Envelope Approximation (SVEA). First the modes are found for the linear part of the refractive index structure. By suitably choosing the complex value of the propagation step, one mode is maximally increased in amplitude. After the nonlinearity has been put on, two methods are applied to find the modes for the nonlinear structure. One method is the same as the method used for the linear part, in the other method the propagation step is left unchanged. The applicability of the method is discussed and illustrated by a calculation on a waveguide with one-dimensional cross section having Kerr-type nonlinearity.
\end{abstract}

\section{INTRODUCTION}

$\mathbf{I}$

$\mathrm{N}$ the last few years, a number of papers have appeared

discussing the propagation of waves in nonlinear media showing third-order nonlinear effects of the intensity dependent refractive index. Special interest has been given to analytical [1]-[5] and numerical [6], [7] methods for finding solutions in multilayer slab waveguides, the nonlinearity being of Kerr-type in most papers, e.g., in [1]-[5].

For linear structures, a number of methods have been developed to find modal field solutions for an arbitrary refractive index profile. Two-dimensional transfer matrix methods can be used for two-dimensional structures [8]-[11], whereas threedimensional structures require other methods. Many methods essentially solve an eigenvalue problem with a matrix having a dimension equal to the number of grid points of the cross section [12]. Another method, by Yevick and Hermansson [13], consists of propagating a field distribution along an imaginary propagation direction (throughout this paper, the propagation direction is along the (in general complex) $z$ axis). The idea is that the guided mode with the highest effective index gets the maximum increase in amplitude during propagation. They use as propagation scheme the Fourier Transform Beam Propagation Method (FTBPM) [14]-[16].

In an earlier paper [17], we described the application to linear structures, both nonabsorbing and absorbing, of

Manuscript received August 16, 1994; revised September 17, 1994.

F. Wijnands is with the Condensed Matter Theory Group, The Blackett Laboratory, Imperial College, London SW7 2BZ, UK.

H. W. M. Hoekstra and R. M. de Ridder are with the MESA Research Institute, University of Twente, The Netherlands.

G. J. M. Krijnen is with the University of Central Florida, Center for Research in Electro-Optics and Lasers, Orlando, FL 32826 USA.

IEEE Log Number 9409831. the Finite Difference Beam Propagation Method (FDBPM) [18]-[20] for solving the modal field equation. The advantage of the FDBPM is its fast convergence. Moreover, the FDBPM gives much more accurate results for large index contrasts (see e.g., [21], [22]).

It is the aim of this paper to show that this numerical method is applicable to materials having an intensity dependent refractive index.

The waveguide structure to be analysed is in general $z$ dependent. Since we are interested in finding the modal fields at a cross section of the structure for a given value of $z$, the given cross section will be extended in the direction of propagation, leading to a $z$-invariant "calculation structure."

The paper is organized as follows. In Section II the method is described. A test example is presented in Section III, followed by a discussion of the results.

\section{THEORY}

\section{A. Linear Structures}

In this subsection, the method for treating the linear case [17] is briefly summarized. We restrict ourselves to the twodimensional case and TE-polarization. The method is applicable in three dimensions as well [23]-[25].

Assuming a time dependence term $e^{i \omega t}$, we have

$$
\nabla^{2} E(x, z)+k_{0}^{2} n^{2}(x, z) E(x, z)=0 .
$$

According to the slowly varying envelope approximation (SVEA), $E(x, z)$ may be written as

$$
E(x, z) \equiv \psi(x, z) e^{-i k_{0} n_{0} z}
$$

where $n_{0}$ is a suitably chosen mean refractive index. Discretise the field, $\psi_{j}^{s} \equiv \psi(s \Delta z, j \Delta x), j=1, \cdots, N$, and introduce the vector $\Psi^{s},\left(\Psi^{s}\right)_{j} \equiv \psi_{j}^{s}$. Substituting (2) in (1), neglecting the term $\partial^{2} \psi / \partial z^{2}$ and integrating the result according to Crank-Nicolson yields [19]:

$$
\left[\mathbf{1}+i \boldsymbol{M} \Delta z /\left(4 k_{0} n_{0}\right)\right] \boldsymbol{\Psi}^{s+1}=\left[\mathbf{1}-i \boldsymbol{M} \Delta z /\left(4 k_{0} n_{0}\right)\right] \boldsymbol{\Psi}^{s} .
$$

Here $\mathbf{1}$ is the identity matrix, $\boldsymbol{M}$ is a tridiagonal matrix with

$$
\begin{array}{r}
\boldsymbol{M}_{i j}=\frac{1}{(\Delta x)^{2}}, \quad \boldsymbol{M}_{i i}=\frac{-2}{(\Delta x)^{2}}+k_{0}^{2}\left(n_{i}^{2}-n_{0}^{2}\right), \\
1 \leq i \leq N, \quad j=i \pm 1, \quad \boldsymbol{M}_{i j}=0
\end{array}
$$

elsewhere. Transparent boundary conditions are used [26], and efficient interface conditions are applied [21]. 
Suppose a structure carries $N$ modal fields $\boldsymbol{\theta}_{0}, \cdots, \boldsymbol{\theta}_{N-1}$ at $z=s \Delta z$, so $\boldsymbol{\Psi}^{s}=\Sigma_{j=0}^{N-1} \alpha_{j} \boldsymbol{\Theta}_{j}$. For each modal field $\boldsymbol{\Theta}_{j}$, we have

$$
\boldsymbol{M} \boldsymbol{\Theta}_{j}=k_{0}^{2}\left(n_{e_{j}}^{2}-n_{0}^{2}\right) \boldsymbol{\theta}_{j}
$$

Here $n_{e_{j}}$ is the effective mode index. Substitution of (4) in (3) yields:

$$
\boldsymbol{\Psi}^{s+1}=\sum_{j=0}^{N-1} \frac{4 \beta_{0}-i \Delta z\left(\beta_{e_{j}}^{2}-\beta_{0}^{2}\right)}{4 \beta_{0}+i \Delta z\left(\beta_{e_{j}}^{2}-\beta_{0}^{2}\right)} \alpha_{j} \boldsymbol{\Theta}_{j}
$$

where $\beta_{e_{j}}=k_{0} n_{e_{j}}$ is the propagation constant of the $j$ th mode, $\beta_{0}=k_{0} n_{0}$ and $k_{0}=2 \pi / \lambda_{0}, \lambda_{0}$ is the wavelength of the light.

By giving $\Delta z$ a value in the complex plane such that $4 \beta_{0}+$ $i \Delta z\left(\beta_{e_{j}}^{2}-\beta_{0}^{2}\right) \sim 0$, the mode $\boldsymbol{\theta}_{j}$ is maximally increased in amplitude. For nonabsorbing structures, $i \Delta z \in \mathbb{R}$ (since $\beta_{e_{j}} \in$ $\mathbf{R}$ ), for absorbing structures $\Delta z \notin \mathbb{R}, i \Delta z \notin \mathbb{R}$ (since $\beta_{e_{j}} \notin \mathbb{R}$ ). To find the guided modes, a method equivalent to the inverse iteration method (IIM) [27] is used. In this method, the guessed eigenvalue of a matrix converges to its exact eigenvalue. This is done by varying $\Delta z$. After each propagation step, the value for the propagation constant follows from the guessed eigenvalue. The procedure is continued until the propagation constant has converged sufficiently. For the calculation of the next mode, the previously found mode is filtered out, and the procedure starts again.

\section{B. Nonlinear Structures}

Consider a field $E(x, z)$ with an intensity dependent refractive index $n\left(x, z ;|E(x, z)|^{2}\right)$. In the particular case of third order (Kerr-type) nonlinearity we have

$$
n(x, z ; E(x, z))=n(x, z ; 0)+n_{2}(x, z)|E(x, z)|^{2},
$$

where $n(x, z ; 0)$ and $n_{2}(x, z)$ are the linear and nonlinear refractive index, respectively. The Kerr-nonlinearity can alternatively be defined with respect to the relative permittivity $\epsilon=n^{2}$ as

$$
\epsilon(x, z ; E(x, z))=\epsilon(x, z ; 0)+\epsilon_{2}(x, z)|E(x, z)|^{2} .
$$

It will be discussed to which extent the two definitions give the same results. For $\epsilon_{2}(x, z)=2 n(x, z ; 0) n_{2}(x, z)$ and $n_{2}(x, z)|E(x, z)|^{2} \ll 1$ the results can be expected to be the same. If not $n_{2}(x, z)|E(x, z)|^{2} \ll 1$ however, the results might differ.

There are various ways to extend the method described in Section II-A to nonlinear structures. It turns out that this procedure is not very suitable for finding solutions for the nonlinear problem. For example in the linear case, in order to find all guided modes, it is essential to filter out modes that have already been found, if one tries to find the next mode. In the nonlinear case this procedure wouldn't work, since the solutions are not orthogonal.

Another method is to find first the modal field solutions for the linear part of the refractive index profile $(n(x, z ; 0)$ in (6)). Then the nonlinearity is turned on adiabatically (say in 10 propagation steps). This method can be expected to be much better.
Now one could turn on the nonlinearity adiabatically and at the same time adjust the eigenvalue (and consequently $\Delta z$ ) according to the inverse iteration method.

For small nonlinearities this method works. But, for example, in the case of coupled waveguides with nearly degenerate effective indices and large nonlinearities, solutions with nearly degenerate indices become mixed in the numerical solution.

A much better algorithm is achieved if the eigenvalue is fixed while the nonlinearity is being increased. Once the nonlinearity has reached its final value, there are two options left. One could continue the inverse iteration method again (i.e., one adjusts $\Delta z$ ) (referred to as method 1), or keep $\Delta z$ fixed (referred to as method 2). For low powers, a linear $\mathrm{TE}_{i}$ mode can be expected to converge to the corresponding nonlinear mode being the same for the two options. For higher powers however, a linear $\mathrm{TE}_{i}$ mode might converge to a nonlinear $\mathrm{TE}_{j}$ mode, $j \neq i$, being different in general for the two options. For method 2, in the transition power regime between two different nonlinear modal fields, the field might converge very slowly.

As indicated above, for a given linear mode and a given power, the two methods discussed above might yield different nonlinear modes. Therefore an algorithm has been implemented using both methods, in order to find as many nonlinear modes as possible. The method described above is useful for nonlinearities of Kerr-type, and for saturable nonlinearities as well. An advantage of our approach is that it is more generally applicable than the analyical approach described in [1]-[5]. For particular cases in which there exists an analytical solution method, such a method is expected to be more efficient than our method. A second advantage of our method is, that it can be used for the case that two or more modes are nearly degenerate. For the linear case the application of our method to the nearly degenerate case has been described in [17]

\section{The Algorithm}

In this subsection the algorithm, based on the theory described earlier in this Section, is sketched.

1) Search for a Linear Mode:

a) First only the linear part of the refractive index profile is taken into account. For given geometry, for given discretization, and for given norm of the field, say $\boldsymbol{\Psi}$, defined as $\sqrt{\sum_{i=1}^{N} \boldsymbol{\Psi}_{i} \boldsymbol{\Psi}_{i}^{*}}$, the starting field is constructed. The five lowest order (i.e., having the fewest nodes) cosine and sine functions with appropriately chosen period with regard to the width of the waveguide, are matched to an exponentially decaying function. This process is not so delicate, the only objective is that the starting field should contain the linear guided modes supported by the linear part of the refractive index profile.

The starting field is normalized with respect to the norm of the field. At the stage when a mode $\boldsymbol{\theta}_{m}$ has been found, the propagation constant $\beta$ is known, so that the power in the mode can be determined as

$$
P=\frac{\beta}{2 \omega \mu_{0}} \int|E(x, z)|^{2} d x \approx \frac{\beta}{2 \omega \mu_{0}} \Delta x \sum_{i=1}^{N}\left(\boldsymbol{\theta}_{m}\right)_{i}\left(\boldsymbol{\theta}_{m}\right)_{i}^{*} .
$$




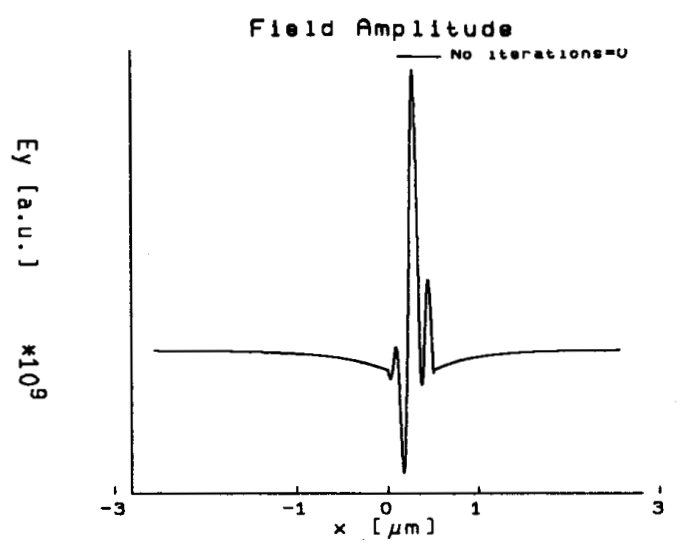

(a)

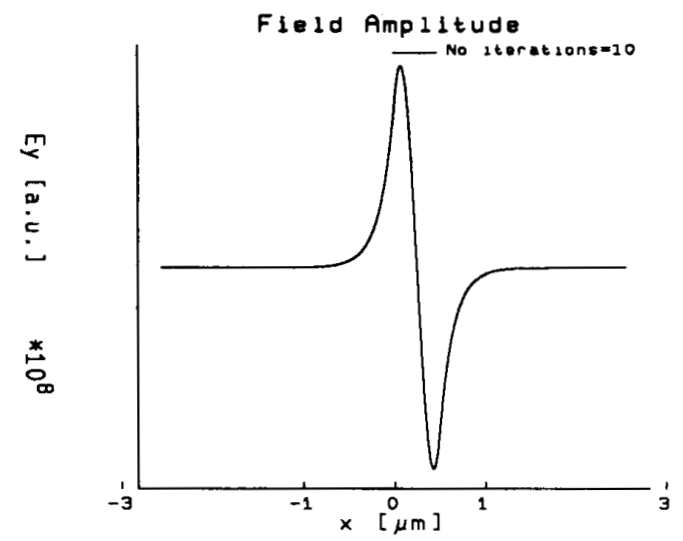

(c)

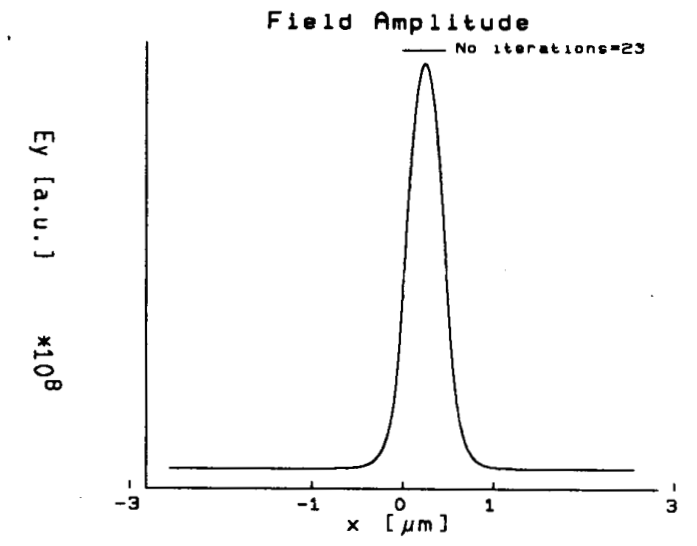

(e)

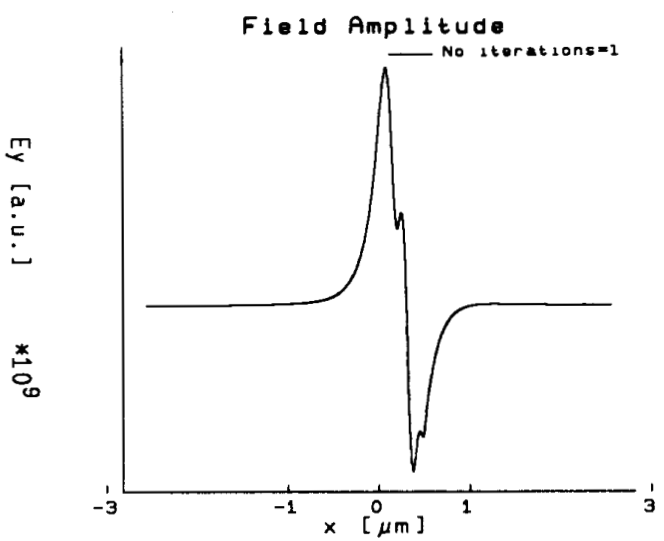

(b)

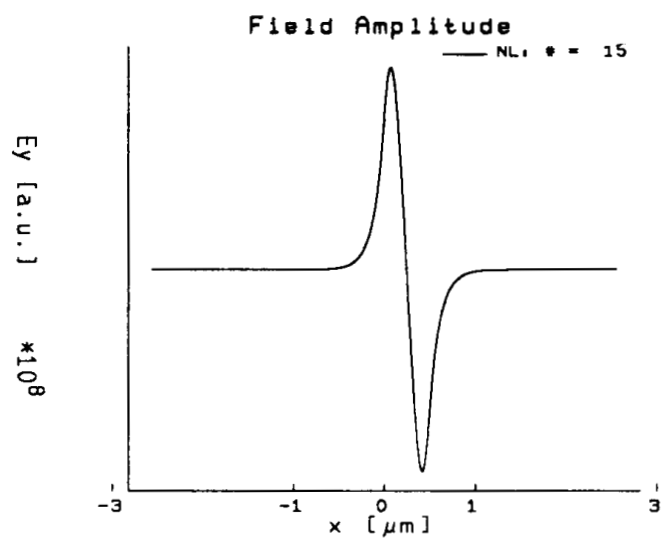

(d)

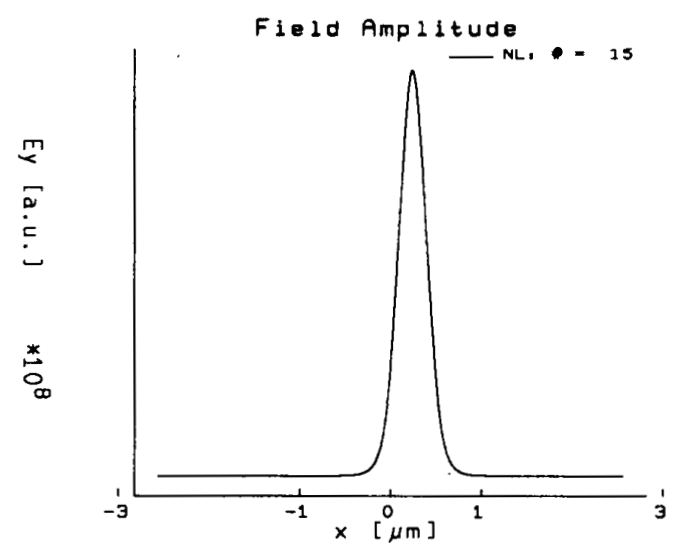

(f)

Fig. 1. Field amplitude after propagation steps for structure in text, starting with a superposition of 5 sines and cosines (a). The linear (nonlinear) $T E_{1,0}$ are shown in (c), (e) ((d), (f)), respectively. Linear modes are found after 10, 23 propagation steps for TE 1,0 respectively. For both modes, in addition, 15 iterations were performed to achieve the nonlinear modes.

b) Now a linear (subscript $L$ ) mode $\mathrm{TE}_{i, L}$ is sought. For each propagation step, a new approximation of the eigenvalue, and consequently of $\Delta z$, is calculated using the IIM (see Section II-A). To each new approximation of $\Delta z$ corresponds a new approximation of the propagation constant $\beta_{e_{i}}$. c) If the difference between two subsequent values of $\beta_{e_{i}}$ is smaller than $10^{-6}$, then the field is decided to have converged to a linear mode $\mathrm{TE}_{i, L}$ with propagation constant $\beta_{e_{i}, L}$ and corresponding $\Delta z_{L}$. Otherwise, the field is normalized with regard to the norm, and we turn to $b$. 
2) Turning on the Nonlinearity:

d) Let the nonlinear part of the refractive index profile be given by $n_{2}$. Then the field resulting from part 1 , is propagated 10 steps with constant $\Delta z_{L}$, with the nonlinearity increased one tenth of $n_{2}$ at each step. After each propagation step, the field is normalized with regard to the norm. After propagating 10 times, we have built up the nonlinear refractive index profile for which we intend to find the nonlinear guided modes.

3) Method 1 for Finding a Nonlinear Guided Mode:

e) After step d, what we have is a field and a value $\Delta z_{L}$, corresponding to an approximation of the eigenvalue. Now exactly the same steps are followed as in steps b and $\mathrm{c}$ : this is just the IIM again. The only difference is that now the refractive index profile is nonlinear instead of linear. As stated in Section II-B, the nonlinear (subscript $N L$ ) mode $\mathrm{TE}_{j, N L}$ can be such that $j \neq i$. As in step c, once the difference between two subsequent values of $\beta_{e_{j}}$ is smaller than $10^{-6}$, the field is decided to have converged to a nonlinear mode $\mathrm{TE}_{j, N L}$ with propagation constant $\beta_{e_{j}, N L}$ and corresponding $\Delta z_{N L}$. The power in the mode is determined using (8).

4) Method 2 for Finding a Nonlinear Guided Mode:

$\left.\mathrm{e}^{\prime}\right)$ After step d, we have a field and a value $\Delta z_{L}$, corresponding to an approximation of the eigenvalue. In this case, the field is propagated with constant value $\Delta z_{L}$, which resulted from step d. After each step, the field is normalized with respect to the norm. Since $\Delta z_{L}$ is fixed now, the propagation constant has to be determined in an alternative way. By propagating the field using a very small real propagation step, the propagation constant is determined from the phase.

Provided the field converges (see discussion in Section IIB), after normalization we end up with a mode $\mathrm{TE}_{k, N L}$ (not necessarily $k=j$ ), once the difference between two subsequent values of $\beta_{e_{k}, N L}$ is smaller than $10^{-6}$. The power in the mode is determined using (8).

5) Convergence Check of Nonlinear Mode:

f) Both for method 1 and 2, once a nonlinear mode has been found, its stability is checked by studying the phase of the field when propagating along a real propagation distance (the usual BPM-algorithm). When the phase, and consequently the effective index, varies too much, the field is considered to be not (yet) converged.

6) What to Do Once a Nonlinear Mode Has Been Found?

g) In order to find the next guided mode, the starting field of step a is orthogonalized with respect to the linear mode found in part 1 . If the norm of the field after orthogonalisation is smaller than a critical value, the field is supposed to contain no more linear guided modes, and the algorithm is stopped. If the norm exceeds this critical value, the field is normalized and steps $b$ and $c$ are followed again with one additional step. Since we don't want to blow up linear modes which have already been found, before the next propagation step the field is orthogonalized with respect to already found linear modes (and then normalized).

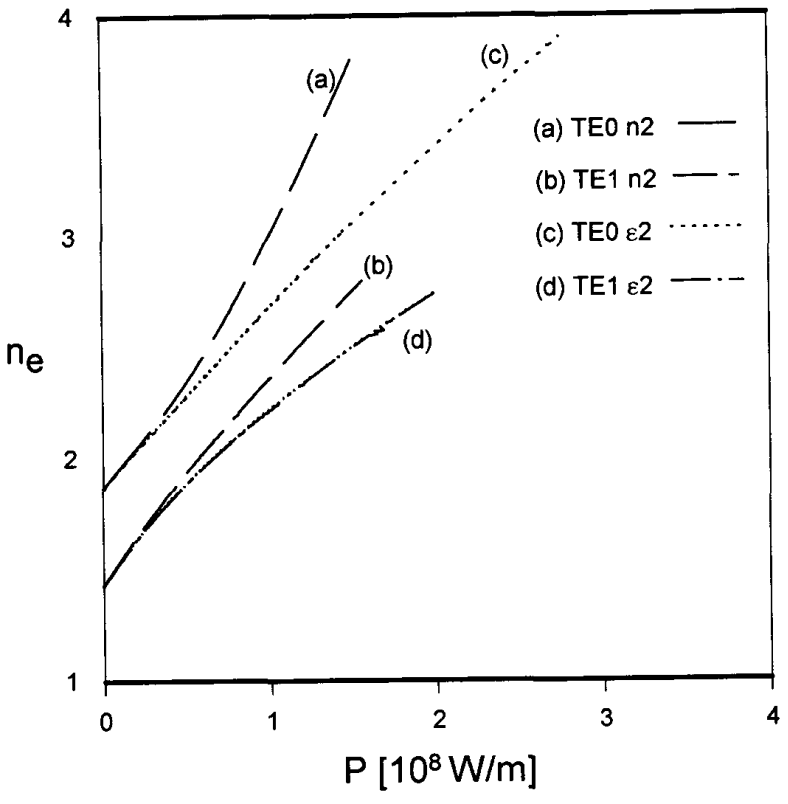

Fig. 2. Effective index $n_{e_{j}}$, as function of the mode power $P(\mathrm{~W} / \mathrm{m})$ for $\mathrm{TE}_{j}, j=0,1$ with nonlinearity with respect to refractive index, $n_{2}=10^{-17} \mathrm{~m}^{2} / \mathrm{V}^{2}$ and with respect to relative permittivity, $\epsilon_{2}=4.010^{-17} \mathrm{~m}^{2} / \mathrm{V}^{2}$, for structure in text.

There is another criterion to check whether no more guided modes will be found. If, in step $b$, the propagation constant drops below a critical value for a number of subsequent propagation steps, we conclude that there are only radiation modes left in the field, and the algorithm is stopped.

\section{EXAMPLE}

In this section, a one-dimensional three-layer example, also described in [3], is treated, with $n_{f}=2.0, n_{c}=n_{s}=$ 1.0 being the linear refractive index of nonlinear film, linear cladding and linear substrate, respectively. The wavelength $\lambda=1.0 \mu \mathrm{m}$, the thickness of the film layer is $0.5 \lambda=$ $0.5 \mu \mathrm{m}$. The transverse grid spacing $\Delta x=0.0075 \mu \mathrm{m}$ and the number of grid points is 2048 .

We first consider positive nonlinearity, with $n_{2}=$ $10^{-17} \mathrm{~m}^{2} / V^{2}, \epsilon_{2}=2 n_{f} n_{2}=4 \times 10^{-17} \mathrm{~m}^{2} / \mathrm{V}^{2}$. We choose $n_{0}=n_{c}$. The convergence criterion will be that successive values of $\beta_{e_{j}}$ should have a difference less than $10^{-9}$.

For zero power, two guided TE-modes are supported. To illustrate how our method works, Fig. 1 shows the field amplitude after some propagation steps, starting with a superposition of the 5 lowest order sine and cosine functions with appropriate period with respect to the film layer thickness, which are matched to an exponentially decaying function. The power is such that the two methods mentioned in Section II-B give convergence to the same nonlinear modal field.

Fig. 2 shows the effective index $n_{e_{j}}$ as function of the total mode power $P$ for $\mathrm{TE}_{j}, j=0,1$, for both definitions of nonlinearity (6) and (7). One would expect that the difference in slope $\partial n_{e_{j}} / \partial P$ for the two definitions of nonlinearity, is 


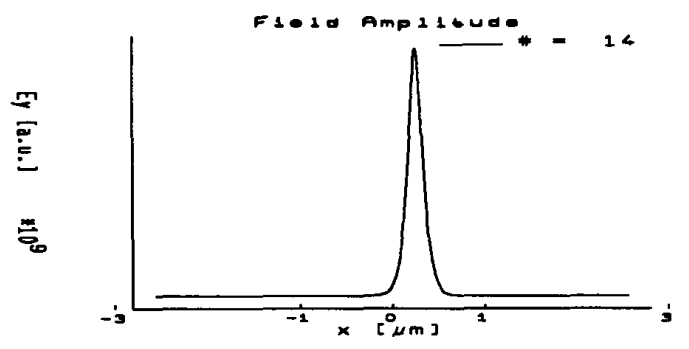

(a)

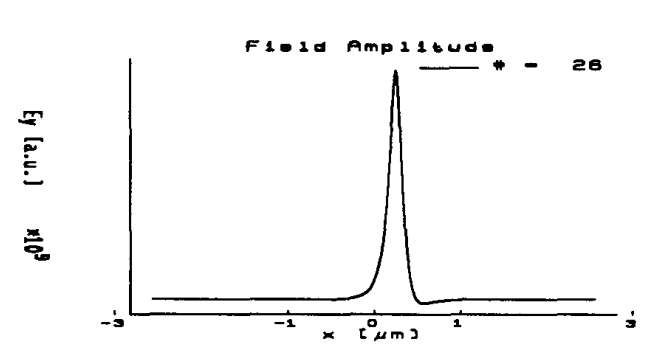

(c)

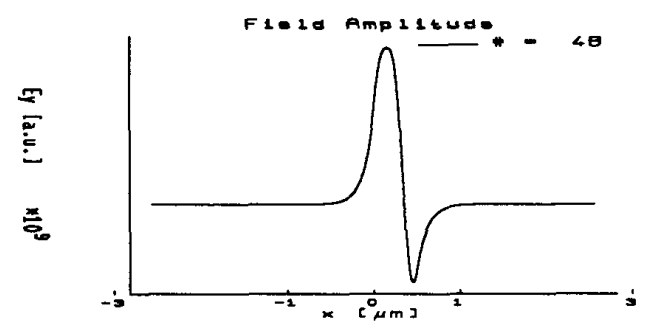

(e)

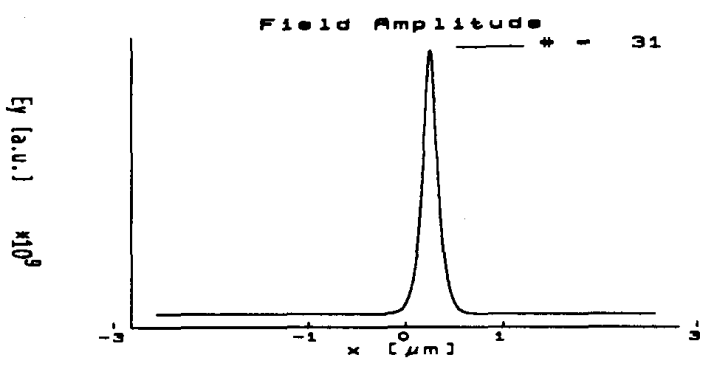

(b)

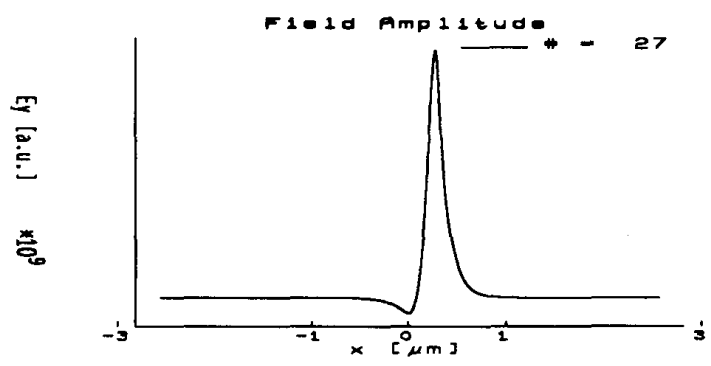

(d)

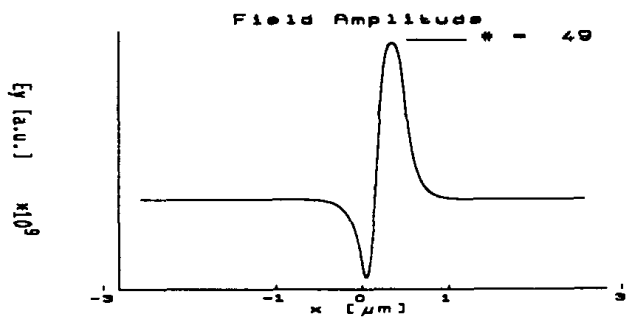

(f)

Fig. 3. Some field amplitudes for nonlinear structure in text for $P \approx 2.510^{7} \mathrm{~W} / \mathrm{m}$ for calculation method 1 after 14, 31 steps ((a), (b)) and for method 2 after $26,27,48,49$ steps ((c), (d), (e), (f)), and for $P \approx 3.010^{7} \mathrm{~W} / \mathrm{m}$ for method 1 after 14,37 steps ((g), (h)) and for method 2 after 20,21 , 44, 45 steps ((i), (j), (k), (1)). Notice that in our implementation, method 1 and method 2 are applied 14 iterations after the nonlinearity has been put on. Hence, the fields in (a) and (g) apply to both methods.

given by the factor $2 n_{f} n_{2} / \epsilon_{2}=1$ for small $P$ (since then one can neglect the highest order term in the square of (6)). Indeed this is observed in Fig. 2.

Notice that if $\epsilon_{2}$ (or $n_{2}$ ) is scaled by an arbitrary positive factor $\kappa$ and $|E(x, z)|^{2}$ by a factor $1 / \kappa$, then the refractive index is left unchanged, and consequently also the effective index. Due to (8), the power will be scaled by a factor $1 / \kappa$. It is shown in Fig. 2 that for low powers the effective index increases linearly with power (notice that we are dealing with positive nonlinearity) consistent with coupled mode theory [4], which predicts for small powers a dependence

$$
n_{e}(P)=n_{e}(0)+\alpha P,
$$

where $\alpha$ is a constant.

For the $\mathrm{TE}_{0}$ mode, for higher powers, the increase is faster than linearly with power if the nonlinearity is defined according to (6), and slower than linearly with power for nonlinearities according to (7). For the $\mathrm{TE}_{1}$ mode, the increase is slower than linearly with power in both cases, but for given $P$, the value is larger in the case of (6), than for (7).
One sees that for both modes, for given $P$, the value for the effective index is larger in case of nonlinearity according to (6). This is due to the fact that for higher powers, the term $n_{2}(x, z)|E(x, z)|^{2}$ becomes larger and hence also the effective index.

For nonlinearities according to (6), this behavior is reflected in Table $\mathrm{I}$, in which the values of $n_{e_{0}}$ for some values of the power achieved with our method are compared to the results of a self-consistent field method [28]. Coupled mode theory predicts for the structure under consideration a value $\alpha=$ $9.059110^{-9} \mathrm{~m} / \mathrm{W}$, whereas Table I gives, for $P=6.1846698$ $\mathrm{W} / \mathrm{m}$, a value $\alpha=9.052710^{-9} \mathrm{~m} / \mathrm{W}$. From Table $\mathrm{I}$ it can be seen that there is nice agreement between our method and the self-consistent field method, also for high powers.

It has already been discussed in Section II-B that for method 2, in the transition power regime between two different nonlinear modal fields, the field might converge very slowly. This is due to the fact that the value for $\Delta z$, needed to blow up a linear mode with a particular value for $n_{e}$, is left unchanged in the case of method 2 when turning to the search for a 


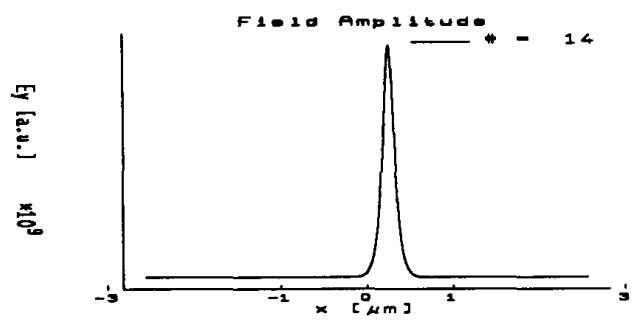

(g)

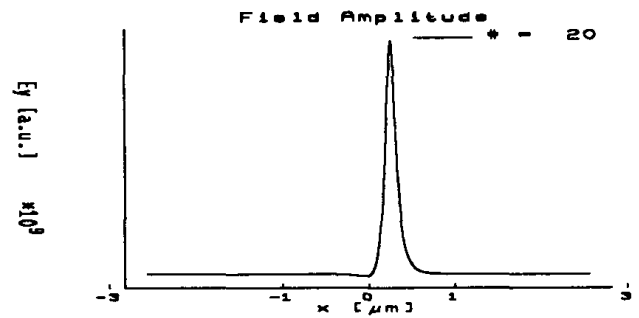

(i)

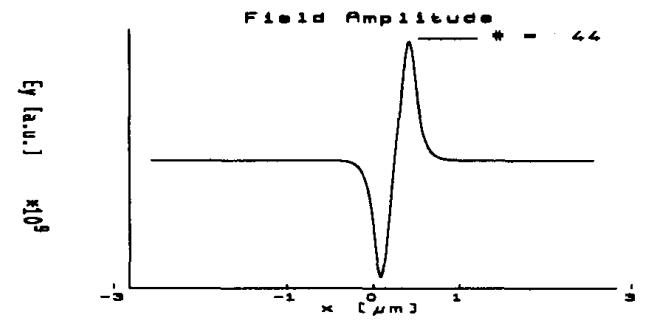

(k)

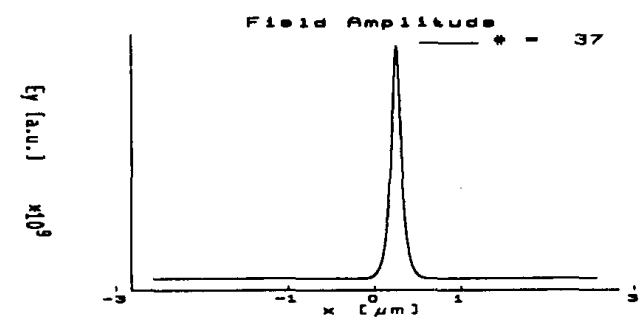

(h)

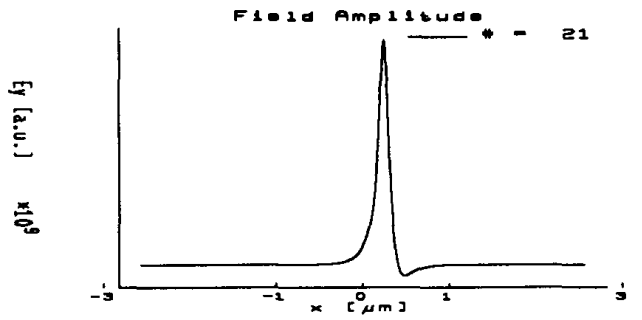

(j)

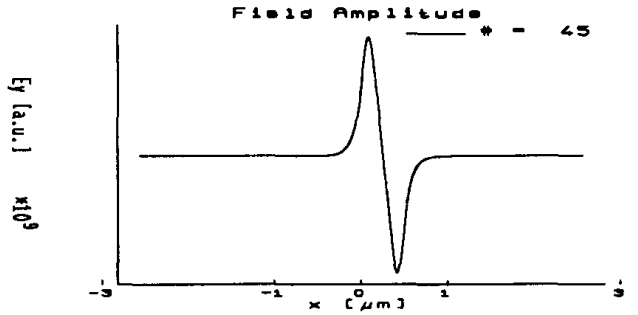

(1)

Fig. 3. (continued) Some field amplitudes for nonlinear structure in text for $P \approx 2.510^{7} \mathrm{~W} / \mathrm{m}$ for calculation method 1 after 14,31 steps ((a), (b)) and for method 2 after $26,27,48,49$ steps $((\mathrm{c}),(\mathrm{d}),(\mathrm{e}),(\mathrm{f}))$, and for $P \approx 3.010^{7} \mathrm{~W} / \mathrm{m}$ for method 1 after 14,37 steps $((\mathrm{g})$, (h)) and for method 2 after 20,21,44, 45 steps ((i), (j), (k), (l)). Notice that in our implementation, method 1 and method 2 are applied 14 iterations after the nonlinearity has been put on. Hence, the fields in (a) and (g) apply to both methods.

TABLE I

EFFective INdex $n_{e_{0}}$ For SOMe VAlues of THE Power $P(\mathrm{~W} / \mathrm{m})$ WITH NONLINEARITY DEFINED WITH RESPECT TO THE REFRACTIVE INDEX $n_{2}=10^{-17} \mathrm{~m}^{2} / \mathrm{V}^{2}$ OF STRUCTURE DESCRIBED IN TEXT.

Also the Values as Calculated with a Self-Consistent Field Method ARE GIVEN IN THE Form $\delta n_{e}(P)=n_{e, \text { new }}(P)-n_{e, \text { self }}(P)$

\begin{tabular}{|c|c|c|}
\hline & new method & self-consistent \\
\hline$P[W / m]$ & $n_{e_{0}}$ & $\delta n_{e}$ \\
\hline 0.0 & 1.86394674 & $-5.1110^{-6}$ \\
\hline $6.184669810^{\circ}$ & 1.86394680 & $-5.1110^{-6}$ \\
\hline $1.012030110^{7}$ & 1.95517590 & $-1.49 \quad 10^{-6}$ \\
\hline $3.156727210^{7}$ & 2.15244357 & $-2.22310^{-5}$ \\
\hline $5.468247410^{7}$ & 2.39191600 & $-2.77910^{-5}$ \\
\hline
\end{tabular}

nonlinear mode. Hence, it might happen for some power $P$ that with this particular $\Delta z$, two different nonlinear modes get nearly the same blow up factor. Restricting ourselves to the definition of nonlinearity (6), we see from Fig. 2 that $P \approx 2.510^{7} \mathrm{~W} / \mathrm{m}$ is in such a transition regime for the linear $\mathrm{TE}_{0}$ mode. To illustrate the different behavior of the two methods, Fig. 3 shows nonlinear fields after some propagation steps for $P \approx 2.510^{7} \mathrm{~W} / \mathrm{m}$ and $P \approx 3.010^{7} \mathrm{~W} / \mathrm{m}$ for both methods. As can be seen from Fig. 3, for both powers, method 1 gives convergence to a nonlinear $\mathbf{T E}_{0}$ mode ((3b) and (3h)). In the case of method 2 however, for $P \approx 2.510^{7} \mathrm{~W} / \mathrm{m}$ the field tends to a $\mathrm{TE}_{1}$ mode without converging (comparing (3e), (3f)). For the slightly increased power $P \approx 3.010^{7} \mathrm{~W} / \mathrm{m}$, the tendency to converge to a $\mathrm{TE}_{1}$ mode is larger (compare (3k) and (31)).

In analyical studies of nonlinear waveguides [1], [3], an important dimensionless parameter is $J$, defined as

$$
J \equiv \epsilon_{2}\left|E_{0}\right|^{2}
$$

where $\epsilon_{2}$ and $\left|E_{0}\right|$ are nonlinearity and field amplitude, respectively, at the two interfaces between nonlinear and linear media. Fig. 4 shows the effective index $n_{e_{j}}$ as function of $J$ for $\mathrm{TE}_{0}(4 \mathrm{a})$ and $\mathrm{TE}_{1}(4 \mathrm{~b})$ for both definitions of nonlinearities (6) and (7). In order to compare the results for the two definitions, in the case of (6), $J$ is defined as

$$
J \equiv 2 n_{f} n_{2}(x, z)\left|E_{0}\right|^{2}=4 n_{2}(x, z)\left|E_{0}\right|^{2} .
$$

Notice that the effective index is left unchanged if $\epsilon_{2}$ (or $n_{2}$ ) is scaled by an arbitrary positive factor $\kappa$ and $|E(x, z)|^{2}$ by a factor $1 / \kappa$. By definition of $J$, the curve of $n_{e}$ as function of $J$, 


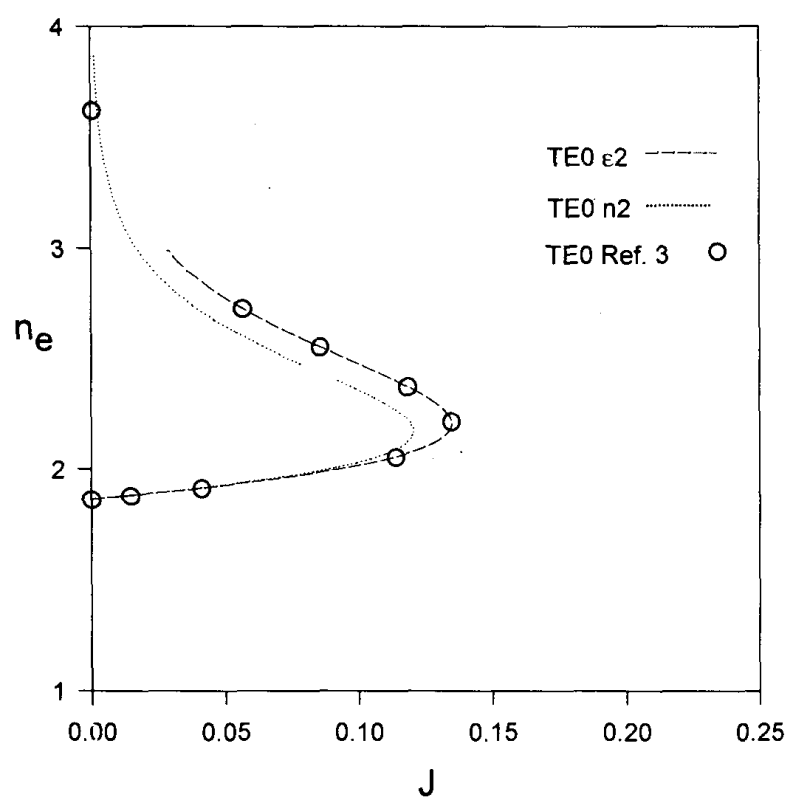

(a)

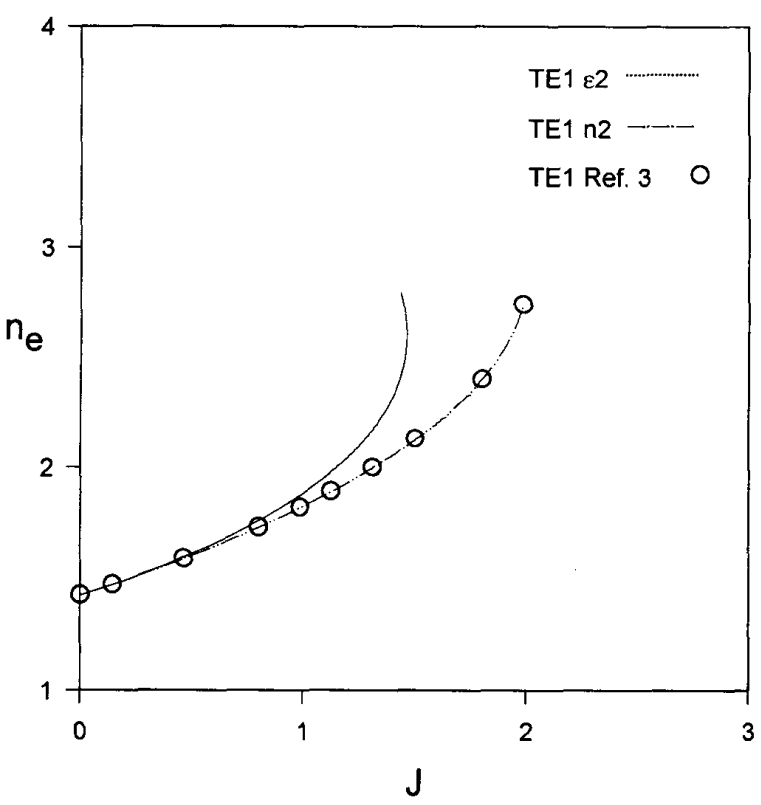

(b)

Fig. 4. Effective index $n_{e_{0}}$, (a) and $n_{e_{1}}$, (b) as function of $J=2 n_{f} n_{2}\left|E_{0}\right|^{2}, n_{2}=10^{-17} \mathrm{~m}^{2} / \mathrm{V}^{2}$, for nonlinearity with respect to refractive index, and as function of $J=\epsilon_{2}\left|E_{0}\right|^{2}, \epsilon_{2}=4.010^{-17} \mathrm{~m}^{2} / \mathrm{V}^{2}$ for nonlinearity with respect to relative permittivity, for structure in text, together with exact results of [3]. Here, the linear refractive index in the film layer is denoted by $n_{f} ; n_{2}, \epsilon_{2}$ and $\left|E_{0}\right|$ denote nonlinearity in refractive index, nonlinearity in relative permittivity, and field amplitude, respectively, at the (lower) interface between nonlinear film and linear cladding for structure in text.

as given in Fig. 4, is exactly the same for every value of $\epsilon_{2}$ (or $n_{2}$ ). The analytic results of Fig. 1 of [3] are plotted in Fig. 4 as well. There is good agreement between [3] and our results for the nonlinearity according to (7). Comparing the results for the

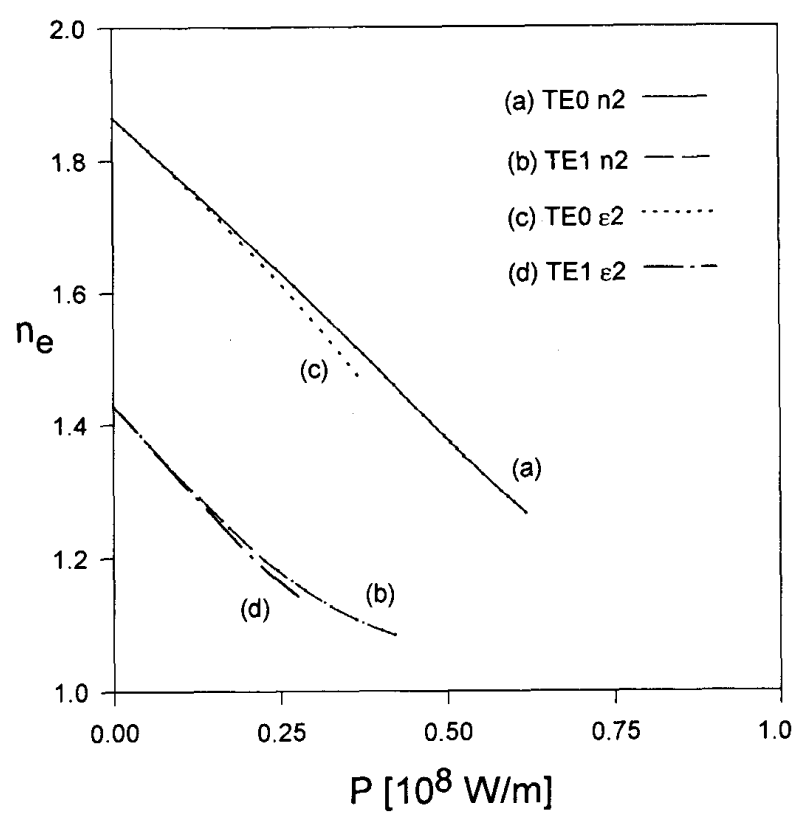

Fig. 5. Effective index $n_{e_{j}}$, as function of the mode power $P(\mathrm{~W} / \mathrm{m})$ for $\mathrm{TE}_{j}, j=0,1$ with nonlinearity with respect to refractive index, $n_{2}=-10^{-17} \mathrm{~m}^{2} / \mathrm{V}^{2}$ and relative permittivity, $\epsilon_{2}=-4.010^{-17} \mathrm{~m}^{2} / \mathrm{V}^{2}$, for structure in text.

nonlinearity according to (6) and (7), one sees that for small effective index changes with respect to the linear case, good agreement is found. In the region where the effective index change is large, the two curves do not coincide. The reason is that the highest order term in the square of (6) can not be neglected anymore in that case. The data for the nonlinear $\mathrm{TE}_{i}$ mode $(i>1)$ have not been plotted. All nonlinear $\mathrm{TE}_{i}$ modes $(i>1)$ found with our method had not converged according to the phase criterion (described in algorithmic step $f$ ) in Section II-C). They were all found using method 2. Method 1 did not have this problem. As already stated, in some regions, method 2 gives very bad convergence. We believe that this situation applies to the badly converging fields for $\mathrm{TE}_{i}, i>1$, as well as to the data in Fig. 2(d) in the high power regime, (the data in this regime have been achieved using method 2) which show some irregularity around $P \approx 1.7510^{8} \mathrm{~W} / \mathrm{m}$.

To our knowledge, no stability criterion is known for the geometric structure under consideration (a nonlinear film bounded by two linear media). For some geometries, a stability criterion has been formulated. For example, for single interface structures, a mode is stable, if and only if $\partial n_{e} / \partial P>0$. This criterion is not valid in general [29].

Next, we consider negative nonlinearity, with $n_{2}=$ $-10^{-17} \mathrm{~m}^{2} / \mathrm{V}^{2}, \epsilon_{2}=2 n_{f} n_{2}=-4 \times 10^{-17} \mathrm{~m}^{2} / \mathrm{V}^{2}$. Fig. 5 shows the effective index $n_{e_{j}}$ as function of the total mode power $P$ for $\mathrm{TE}_{j}, j=0,1$, for the nonlinearity according to (6) and (7). For the nonlinearity according to (6), the value of $n_{e}$ at $P=12.369 \mathrm{~W} / \mathrm{m}$ leads to the value $\alpha=-9.053410^{-9} \mathrm{~m} / \mathrm{W}$ for the proportionality constant in (9). For higher powers, the decrease is slower than linearly with power for both definitions of nonlinearity in case of $\mathrm{TE}_{1}$. For $\mathrm{TE}_{0}$, the decrease is faster than linearly with power for nonlinearity according to (7). 


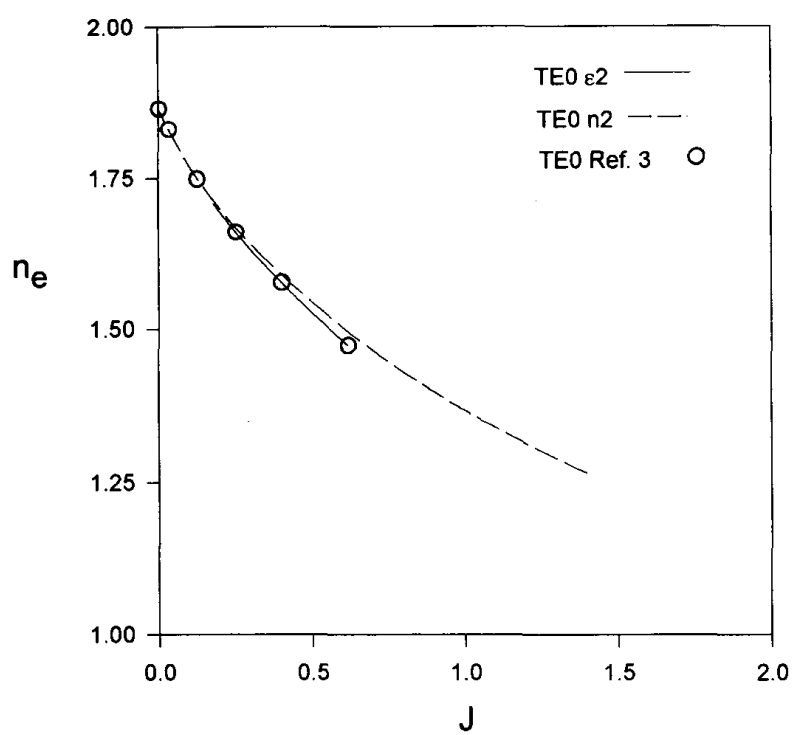

(a)

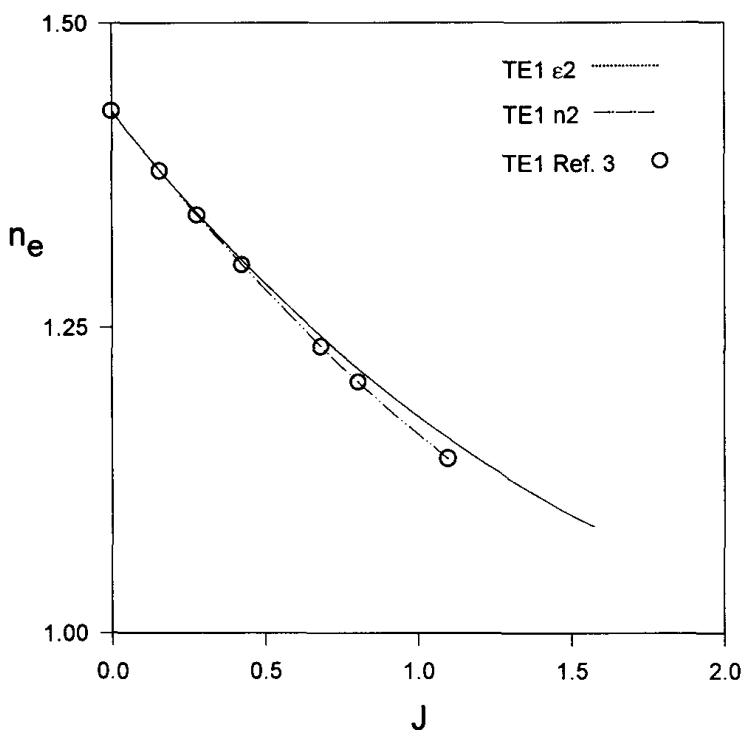

(b)

Fig. 6. Effective index $n_{e_{0}}$, (a) and $n_{e_{1}}$, (b) as function of $J=2 n_{f} n_{2}\left|E_{0}\right|^{2}, n_{2}=-10^{-17} \mathrm{~m}^{2} / \mathrm{V}^{2}$, for nonlinearity with respect to refractive index, and as function of $J=\epsilon_{2}\left|E_{0}\right|^{2}, \epsilon_{2}=-4.010^{-17} \mathrm{~m}^{2} / \mathrm{V}^{2}$ for nonlinearity with respect to relative permittivity, for structure in text, together with exact results of [3]. Here the linear refractive index in the film layer is denoted by $n_{f} ; n_{2}, \epsilon_{2}$ and $\left|E_{0}\right|$ denote nonlinearity in refractive index, nonlinearity in relative permittivity, and field amplitude respectively, at the (lower) interface between nonlinear film and linear cladding for structure in text.

Fig. 6 shows the effective index $n_{e_{j}}$ as function of $J$ for $\mathrm{TE}_{0}(6 \mathrm{a})$ and $\mathrm{TE}_{1}(6 \mathrm{~b}), J$ being defined as in (11), (10) for (6), (7), respectively, together with the analytic results of Fig. 5 of [3]. There is good agreement between [3] and our results for the nonlinearity according to (7). Comparing the results for the nonlinearity according to (6) and (7), one sees again that for small effective index changes with respect to the linear case, good agreement is found. In the region where the effective index change is large, the two curves do not coincide. The two asymmetrical modes presented in Fig. 5 of [3], have not been found with our method.

For positive nonlinearity, in the present implementation, all linear modes converge to very sharply peaked nonlinear modes for high powers. We believe that these solutions are soliton-like.

\section{CONCLUSION}

A nonlinear modal fields calculation method has been applied to the Finite Difference BPM scheme. A calculation scheme has been discussed to find stable nonlinear modal fields for a given power. The numerical results for third order nonlinearity defined with respect to refractive index and relative permittivity have been compared. In the latter case comparison with exact results shows excellent agreement. If the effective index change due to the nonlinearity is large, the two definitions of nonlinearity yield a different behavior.

\section{ACKNOWLEDGMENT}

It is a pleasure to thank R. Varshney and T. Rasmussen for fruitful discussions, and A. Bakker for the software support.

\section{REFERENCES}

[1] A. D. Boardman and P. Egan, "Optically nonlinear waves in thin films," J. Quantum Electron., vol. QE-22, no. 2, pp. 319-324, 1986.

[2] N. N. Akhmediev, K. O. Boltar, and V. M. Eleonskii, "Dielectric optical waveguide with nonlinear susceptibility. Asymmetric refractive-index profile," Opt. Spectrosc. (USSR), vol. 6, no. 53, pp. 654-658, 1982.

[3] W. Chen and A. A. Maradudin, " $s$-polarized guided and surface electromagnetic waves supported by a nonlinear dielectric film," J. Opt. Soc. Am. B, vol. 5, no. 2 , pp. 529-538, 1988.

[4] C. T. Seaton, X. Mai, G. I. Stegeman, and H. G. Winful, "Nonlinear guided wave applications," Optical Engineering, vol. 24, no. 4, pp. $593-599,1985$

[5] L. Leine, C. Wächter, U. Langbein, and F. Lederer, "Evolution of nonlinear guided optical fields down a dielectric film with a nonlinear cladding," J. Opt. Soc. Am. B, vol. 5, no. 2, pp. 547-558, 1988.

[6] F. Dios, L. Torner and F. Canal, "Self-consistent solution for general nonlinear slab waveguides," Opt. Commun., vol. 72, nos. 1 and 2, pp. 54-59, 1989.

[7] M. R. Ramadas, R, K. Varshney, K. Thyagarajan, and A. K. Ghatak, "A matrix approach to study the propagation characteristics of a general nonlinear planar waveguide," J. Lightwave Technol., vol. 7, no. 12, pp. $1901-1904,1989$

[8] A. Ghatak, K. Thyagarajan, and M. R. Shenoy, "Numerical analysis of planar optical waveguides using matrix approach," $J$. Lightwave Technol., vol. LT-5, no. 5, pp. 660-667, 1987.

[9] J. Chilwell and I. Hodgkinson, "Thin-films field-transfer matrix theory of planar multilayer waveguides and reflection from prism-loaded waveguides," J. Opt. Soc. Am. A, vol. 1, no. 7, pp. 742-753, 1984.

[10] K.-H. Schlereth and M. Tacke, "The complex propagation constant of multilayer waveguides: An algorithm for a personal computer," $J$. Ouantum Electron., vol. 26, no. 4, pp. 627-630, 1990.

[11] R. E. Smith, S. N. Houde-Walter, and G. W. Forbes, "Numerical determination of planar waveguide modes using the analyticity of the dispersion relation," Opt. Lett., vol. 16, no. 17, pp. 1316-1318, 1991.

[12] Working group 1, COST 216, "Comparison of different modelling techniques for longitudinally invariant integrated optical waveguides," Proc. IEEE, vol. 136, Pt. J, no. 5, pp. 273-280, 1989 and references therein.

[13] D. Yevick and B. Hermansson, "New formulations of the matrix beam propagation method: Application to rib waveguides," J. Quantum Electron., vol. 25, pp. 221-229, 1989.

[14] J. van Roey, J. van der Donk, and P. E. Lagasse, "Beam-propagation method: Analysis and assessment," J. Opt. Soc. Amer., vol. 71, no. 7 . pp. $423-429,1981$. 
[15] L. Thylen, "The beam propagation method: An analysis of its applicability," Opt. Quantum Electron., vol. 15, pp. 433-439, 1983.

[16] P. E. Lagasse and R. Baets, "Application of propagation beam methods to electromagnetic and acoustic wave propagation problems: A review," Radio Sci., vol. 7, no. 22, pp. 1225-1233, 1987.

[17] F. Wijnands, H. J. W. M. Hoekstra, G. J. M. Krijnen, and R. M. de Ridder, "Modal fields calculation using the finite difference beam propagation method," J. Lightwave Technol., vol. 12, no. 12, pp. 2066-2072, Dec. 1994.

[18] D. Yevick and B. Hermansson, "Efficient beam propagation techniques," J. Quantum Electron., vol. 26, pp. 109-112, 1990.

[19] Y. Chung and N. Dagli, "An assessment of finite difference beam propagation method," IEEE J. Quantum Electron., vol. 26, no. 8, pp. 1335-1339, 1990.

[20] R. Accornero, M. Artiglia, G. Coppa, P. di Vita, G. Lapenta, M. Potenza, and P. Ravetto, "Finite difference methods for the analysis of integrated optical waveguides," Electron. Lett., vol. 26, no. 23, pp. 1959-1960, 1990.

[21] H. J. W. M. Hoekstra, G. J. M. Krijnen, and P. V. Lambeck, "Efficient interface conditions for the finite difference beam propagation method," J. Lightwave Technol., vol. 10, no. 10, pp. 1352-1355, 1992

[22] "New formulation of the beam propagation method based on the slowly varying envelope approximation," Opt. Commun., vol. 97, pp. 301-303, 1993.

[23] F. Wijnands, T. Rasmussen, H. J. W. M. Hoekstra, J. H. Povlsen, A. Bjarklev, and R. M. de Ridder, "Efficient interface conditions for the semi-vectorial Finite Difference Beam Propagation Method," submitted to Opt. and Quantum Electron.

[24] F. Wijnands, T. Rasmussen, H. J. W. M. Hoekstra, J. H. Povlsen, and R. M. de Ridder, "Efficient semi-vectorial mode solvers," submitted to J. Lightwave Technol.

[25] T. Rasmussen, F. Wijnands, H. J. W. M. Hoekstra, J. H. Povlsen, P. G. Dinesen, P. Krishnaswamy, A. Bisgaard, J. Øster, and R. M. de Ridder, "Optimization of the nonuniform grid finite difference method for optical waveguides," to be submitted.

[26] G. R. Hadley, "Transparent boundary condition for beam propagation," Opt. Lett., vol. 16, no. 9, pp. 624-626, 1991.

[27] W. H. Press, B. P. Flannery, S. A. Teukolsky, and W. T. Vetterling, Numerical Recipes. Cambridge: Cambridge Univ. Press, 1986, pp. $377-380$.

[28] G. J. M. Krijnen, H. J. W. M. Hoekstra, and P. V. Lambeck, "A new method for the calculation of propagation constants and field profiles of guided modes of nonlinear channel waveguides based on the effective index method," J. Lightwave Technol., vol. 12, no. 9, pp. 1550-1559, 1994.

[29] A. D. Boardman, P. Egan, F. Lederer, U. Langbein, and D. Mihalache, "Third-order nonlinear electromagnetic TE and TM guided waves," in Nonlinear Surface Electromagnetic Phenomena, H.-E. Ponath and G. I. Stegeman, Eds. Amsterdam: North-Holland, 1991, pp. 73-288.
Frank Wijnands was born in 1962. He received the M.Sc. degree in 1988 and the Ph.D. degree in 1992 both at the University of Nijmegen, the Netherlands, on electronic and crystallographic properties of quasiperiodic crystals.

In 1993, he joined the Lightwave Devices Group at the MESA Research Institute, University Twente, Enschede, The Netherlands, to work on mathematical principles and numerical implementation of beam propagation methods in linear and nonlinear integrated optics. In 1995, he joined the Condensed Matter Theory Group at Imperial College, London, where he is working on photonic band structure of nanostructures.

Hugo J. W. M. Hoekstra was born in 1949 . He received the M.Sc. degree in experimental physics from the University of Amsterdam in 1977. After being a teacher in high school, he began a Ph.D. study on magneto optical properties of solid transition-metal halides at the University of Groningen where he received the Ph.D. degree in 1984.

Between 1984 and 1988, he was a Postdoctoral Fellow in the field of surface science at the Universities of Nijmegen and Groningen. In 1988 he joined the Lightwave Devices Group of the MESA Research Institute at the University of Twente, Enschede, the Netherlands, where he is engaged in work on linear and nonlinear integrated optics.

Gijs J. M. Krijnen (S'90-M'93) started his Ph.D. work in the Lightwave Devices Group of the MESA Research Institute at the University of Twente, Enschede, The Netherlands, in 1987, after receiving the M.Sc. degree in the field of magnetic recording at the Philips Laboratories, Eindhoven, The Netherlands. His Ph.D. research concerned integrated nonlinear optical switching devices.

After finishing his Ph.D. work in May 1992 he started work as a Postdoctoral Fellow of the Royal Netherlands Academy of Arts and Sciences. In this current work his main interest is in the field of the application of nonlinear integrated optics devices as sensors.

René M. de Ridder was born April 26, 1950 in Amsterdam, The Netherlands. $\mathrm{He}$ received the masters degree in 1978 and a $\mathrm{Ph} . \mathrm{D}$. degree in 1988, both in electrical engineering, from the University of Twente, Enschede, The Netherlands.

Since 1981, he has been employed as a lecturer and research scientist at the University of Twente, where he is now a member of the Lightwave Devices Group in the MESA Research Institute. His current research interests include integrated-optic devices for optical communications. 